International Mathematical Forum, 1, 2006, no. 6, 291-298

\title{
Parametric generalized strongly nonlinear mixed quasivariational inclusions in Banach spaces
}

\author{
Jae Ug Jeong \\ Department of Mathematics \\ Dongeui University \\ Pusan 614-714, Korea \\ jujeong@deu.ac.kr
}

\begin{abstract}
In this paper, by using the concept of the resolvent operator, we study the behavior and sensitivity analysis of the solution set for a class of parametric generalized strongly nonlinear mixed quasivariational inclusion problem in Banach spaces. The results presented in this paper are new and generalize many known results in this field.
\end{abstract}

Mathematics Subject Classification: 49J40, 90C20

Keywords: Generalized variational inclusions, Fixed points, Resolvent operator, Sensitivity analysis, Banach spaces.

\section{Introduction}

In recent years, variational inequalities have been extended and generalized in different directions using novel and innovative techniques both for its own sake and for its applications. A useful and important generalization of variational inequalities is called variational inclusions.

In 1994, using the concept and technique of resolvent operators, Hassouni and Moudafi[9] introduced and studied a class of mixed type variational inequalities with single-valued mappings which was called variational inclusions. Adly[1], Ding[7], Huang[10], Kazmi[11], Noor[15] and Noor, Noor and Rassias[16] have obtained some important extensions and generalizations of the results in [9] from various different directions.

Sensitivity analysis of solutions of variational inequalities with single-valued mappings have been studied by many authors via quite different techniques. By using the projection method, Dafermos[6], Yen[20], Mukherjee and Verma[13], Noor[14] and Pan[18] studied the sensitivity analysis of solutions of some variational inequalities with single-valued mappings in finite-dimensional spaces and Hilbert spaces. In 1999, Ding and Luo[8] studied the behavior and sensitivity 
analysis of the solution set for a class of parametric generalized quasivariational inequalities with set-valued mappings by using the projection method of Dafermos[6] in a Hilbert space. The projection method cannot be used to study the behavior and sensitivity analysis of the solution set for variational inequalities with nonlinear term. By using the concept of the resolvent operator, Park and Jeong[19], Agarwal, Cho and Huang[2] and Noor and Noor[17] dealt with the behavior and sensitivity analysis of solutions for parametric variational inequalities(inclusions) with nonlinear terms.

In this paper, we study the behavior and sensitivity analysis of the solution set for a class of parametric generalized strongly nonlinear mixed quasivariational inclusion problem in Banach spaces. The results presented in this paper generalize and improve the corresponding results of $[2,6,17]$.

\section{Preliminaries}

Let $E$ be a real Banach space, $E^{*}$ be the topological dual space of $E,\langle\cdot, \cdot\rangle$ be the dual pair between $E$ and $E^{*}$, and $J: E \rightarrow 2^{E^{*}}$ be the normalized duality mapping defined by

$$
J(x)=\left\{f \in E^{*}:<x, f>=\|x\|\|f\|,\|f\|=\|x\|\right\}, \quad x \in E .
$$

Definition 2.1.[3] Let $M: D(M) \subset E \rightarrow 2^{E}$ be a set-valued mapping.

(1) The mapping $M$ is said to be accretive if, for any $x, y \in D(M), u \in M(x)$, $v \in M(y)$, there exists $j(x-y) \in J(x-y)$ such that

$$
<u-v, j(x-y)>\geq 0 .
$$

(2) The mapping $M$ is said to be m-accretive if $M$ is accretive and $(I+$ $\rho M)(D(M))=E$ for every $\rho>0$, where $I$ is the identity mapping.

We consider now the parametric generalized strongly nonlinear mixed quasivariational inclusion problem in Banach spaces. To this end, let $\Omega$ be a nonempty open subset of $E$ in which the parameter $\lambda$ takes values, $N$ : $E \times E \times \Omega \rightarrow E$ and $M: E \times E \times \Omega \rightarrow 2^{E}$ be an m-accretive mapping with respect to the first argument. For each fixed $\lambda \in \Omega$, the parametric generalized strongly nonlinear mixed quasivariational inclusion problem in Banach spaces consists of finding $u \in E$ such that

$$
0 \in N(u, u, \lambda)+M(u, u, \lambda) .
$$

We now discuss some special cases.

Case I. Let $E=H$ be a Hilbert space, $\Omega$ be a nonempty open subset of $H$ in which the parameter $\lambda$ takes values. Let $\phi: H \times H \times \Omega \rightarrow R \cup\{+\infty\}$ be a functional such that $\partial \phi(\cdot, u, \lambda): H \rightarrow R \subset\{+\infty\}$ denotes the subdifferential of a proper convex lower semicontinuous function $\phi(\cdot, u, \lambda)$. Let $M(\cdot, u, \lambda)=$ $\partial \phi(\cdot, u, \lambda)$ for each $(u, \lambda) \in H \times \Omega$. Then problem (2.1) is equivalent to finding $u \in H$ such that 


$$
<N(u, u, \lambda), v-u>\geq \phi(u, u, \lambda)-\phi(v, u, \lambda), \forall v \in H,
$$

which is called the parametric generalized strongly nonlinear mixed quasivariational inequality problem.

Case II. If $N(u, v, \lambda)=T(u, \lambda)+S(v, \lambda)$ for each $(u, v, \lambda) \in H \times H \times \Omega$, where $S, T: H \times \Omega \rightarrow H$ are two nonlinear mappings, then problem (2.1) is equivalent to finding $u \in H$ such that

$$
0 \in T(u, \lambda)+S(u, \lambda)+M(u, u, \lambda),
$$

which is called the parametric generalized nonlinear mixed quasivariational inclusion problem.

Case III. If $S=0$, then problem (2.2) is equivalent to finding $u \in H$ such that

$$
0 \in T(u, \lambda)+M(u, u, \lambda)
$$

which is called the parametric quasi-variational inclusion problem (see [17]).

Summing up the above arguments, it shows that, for a suitable choice of the mappings $N, M$, we can obtain a number of known and new classes of parametric variational inequalities, parametric variational inclusions, and the corresponding optimization problems from the parametric generalized strongly nonlinear mixed quasivariational inclusion problem in Banach spaces (2.1).

If $M: D(M) \subset E \rightarrow 2^{E}$ is an m-accretive mapping, then for a constant $\rho>0$, the resolvent operator associated with $M$ is defined by

$$
R_{\rho}^{M}(u)=(I+\rho M)^{-1}(u) \text { for all } u \in D(M),
$$

where $I$ is the identity operator. It is well known that $R_{\rho}^{M}$ is a single-valued and nonexpansive mapping $([3])$.

Remark 2.1. Since $M$ is an m-accretive mapping with respect to the first argument, for any fixed $(y, \lambda) \in E \times \Omega$, we define

$$
R_{\rho}^{M(\cdot, y, \lambda)}(u)=(I+\rho M(\cdot, y, \lambda))^{-1}(u), \quad \forall u \in D(M),
$$

which is called the implicit resolvent operator associated with $M(\cdot, y, \lambda)$.

Remark 2.2. It is well known that if $E=E^{*}=H$ is a Hilbert space, then the notion of an accretive mapping coincides with that of a monotone mapping[3].

Remark 2.3. It is also well known if the single-valued operator $M: H \rightarrow H$ is maximal strongly monotone with constant $\alpha>0$, then the resolvent operator $R_{\rho}^{M}=(I+\rho M)^{-1}$ is Lipschitz continuous with constant $\frac{1}{1+\alpha \rho}$ where $\rho>0$ is a constant $[12]$. 
Definition 2.2. Let $N: E \times E \times \Omega \rightarrow E$ be a single-valued mapping. The mapping $N$ is said to be $\beta$-Lipschitz continuous in the first argument if there exists a constant $\beta>0$ such that

$$
\left\|N\left(u_{1}, v, \lambda\right)-N\left(u_{2}, v, \lambda\right)\right\| \leq \beta\left\|u_{1}-u_{2}\right\|, \forall\left(u_{1}, u_{2}, v, \lambda\right) \in E \times E \times E \times \Omega .
$$

In a similar way, we can define the $\xi$-Lipschitz continuity of $N(u, v, \lambda)$ in the second argument.

Lemma 2.1.[4,5] Let $E=L_{p}, p \geq 2$, and $x, y \in E$. Then

$$
\|x+y\|^{2} \leq(p-1)\|x\|^{2}+\|y\|^{2}+2<x, j(y)>.
$$

For the rest of this paper, $E=L_{p}, p \geq 2$, and the single-valued duality map is denoted by $j$.

\section{Main Results}

We first transfer the problem (2.1) into a parametric fixed point theorem.

Theorem 3.1. Fixed $\lambda \in \Omega, u \in E$ is a solution of problem (2.1) if and only if for some given $\rho>0$, the set-valued mapping $F: E \times \Omega \rightarrow E$ defined by

$$
F(u, \lambda)=R_{\rho}^{M(\cdot, u, \lambda)}(u-\rho N(u, u, \lambda))
$$

has a fixed point $u$.

Proof. For each fixed $\lambda \in \Omega$, let $u \in E$ be a solution of the problem (2.1). Then we have

$$
0 \in N(u, u, \lambda)+M(u, u, \lambda)
$$

and hence for any given $\rho>0$,

$$
0 \in-u+\rho N(u, u, \lambda)+u+\rho M(u, u, \lambda) .
$$

By the definition of $R_{\rho}^{M(\cdot, u, \lambda)}$, we have

$$
\begin{aligned}
u & =R_{\rho}^{M(\cdot, u, \lambda)}(u-\rho N(u, u, \lambda)) \\
& =F(u, \lambda),
\end{aligned}
$$

i.e., $u$ is a fixed point of $F$.

Now, let $u$ be a fixed point of $F$ for each fixed $\lambda \in \Omega$. Then

$$
\begin{aligned}
u & =F(u, \lambda) \\
& =R_{\rho}^{M(\cdot, u, \lambda)}(u-\rho N(u, u, \lambda)) .
\end{aligned}
$$

By the definition of $R_{\rho}^{M(\cdot, u, \lambda)}$, we have 


$$
u-\rho N(u, u, \lambda) \in(I+\rho M(\cdot, u, \lambda))(u)
$$

and hence

$$
0 \in N(u, u, \lambda)+M(u, u, \lambda) .
$$

Thus, $u \in E$ is a solution of the problem (2.1).

Theorem 3.1 implies that the parametric generalized strongly nonlinear mixed quasivariational inclusion in Banach space (2.1) and the fixed point problem (3.1) are equivalent. We use this equivalence to study the sensitivity analysis of the problem (2.1).

Theorem 3.2. Let $N: E \times E \times \Omega \rightarrow E$ be a nonlinear mapping such that $N$ is $\alpha$-strongly accretive and $\beta$-Lipschitz continuous in the first argument, and $\xi$-Lipschitz continuous in the second argument. Let $M: E \times E \times \Omega \rightarrow 2^{E}$ be such that for each fixed $(u, \lambda) \in E \times \Omega, M(\cdot, u, \lambda): E \rightarrow 2^{E}$ is an m-accretive mapping. Suppose that for any $(u, v, z, \lambda) \in E \times E \times E \times \Omega$,

$$
\left\|R_{\rho}^{M(\cdot, u, \lambda)}(z)-R_{\rho}^{M(\cdot, v, \lambda)}(z)\right\| \leq \mu\|u-v\|
$$

and there exists a constant $\rho>0$ such that

$$
\begin{gathered}
\left|\rho-\frac{\alpha-\xi(1-\mu)}{(p-1) \beta^{2}-\xi^{2}}\right|<\frac{\sqrt{[\alpha-\xi(1-\mu)]^{2}-\left[(p-1) \beta^{2}-\xi^{2}\right] \mu(2-\mu)}}{(p-1) \beta^{2}-\xi^{2}} \\
\alpha>\xi(1-\mu)+\sqrt{\left[(p-1) \beta^{2}-\xi^{2}\right] \mu(2-\mu)} \\
(p-1) \beta^{2}>\xi^{2}, \quad \mu+\rho \xi<1 .
\end{gathered}
$$

Then

(i) the mapping $F: E \times \Omega \rightarrow E$ defined by (3.1) is a uniform $\theta$-contractive mapping with respect to $\lambda \in \Omega$, where $\theta=\sqrt{1-2 \rho \alpha+(p-1) \rho^{2} \beta^{2}}+\rho \xi+\mu<$ 1.

(ii) for each $\lambda \in \Omega$, the problem (2.1) has a nonempty solution set $S(\lambda)$ and $S(\lambda)$ is a closed subset in $E$.

Proof. (i) By the definition of $F$, for any $(u, \lambda),(v, \lambda) \in E \times \Omega$,

$$
F(u, \lambda)=R_{\rho}^{M(\cdot, u, \lambda)}(u-\rho N(u, u, \lambda)),
$$

and

$$
F(v, \lambda)=R_{\rho}^{M(\cdot, v, \lambda)}(v-\rho N(v, v, \lambda)) .
$$

It follows from (3.2) that 


$$
\begin{aligned}
\| & F(u, \lambda)-F(v, \lambda) \| \\
\leq & \left\|R_{\rho}^{M(\cdot, u, \lambda)}(u-\rho N(u, u, \lambda))-R_{\rho}^{M(\cdot, u, \lambda)}(v-\rho N(v, v, \lambda))\right\| \\
& +\left\|R_{\rho}^{M(\cdot, u, \lambda)}(v-\rho N(v, v, \lambda))-R_{\rho}^{M(\cdot, v, \lambda)}(v-\rho N(v, v, \lambda))\right\| \\
\leq & \|u-v-\rho(N(u, u, \lambda)-N(v, v, \lambda))\|+\mu\|u-v\| \\
\leq & \|u-v-\rho(N(u, u, \lambda)-M(v, u, \lambda))\|+\rho\|N(v, u, \lambda)-N(v, v, \lambda)\| \\
& +\mu\|u-v\| .
\end{aligned}
$$

Since $N$ is $\alpha$-strongly accretive and $\beta$-Lipschitz continuous in the first argument, we have from Lemma 2.1

$$
\begin{aligned}
& \|u-v-\rho(N(u, u, \lambda)-N(v, u, \lambda))\|^{2} \\
& \leq(p-1) \rho^{2}\|N(u, u, \lambda)-N(v, u, \lambda)\|^{2}+\|u-v\|^{2} \\
& \quad-2 \rho<N(u, u, \lambda)-N(v, u, \lambda), j(u-v)> \\
& \leq\left(1-2 \rho \alpha+(p-1) \rho^{2} \beta^{2}\right)\|u-v\|^{2} .
\end{aligned}
$$

Using $\xi$-Lipschitz continuity of $N$ in the second argument, we have

$$
\|N(v, u, \lambda)-N(v, v, \lambda)\| \leq \xi\|u-v\| .
$$

By (3.4)-(3.6), we obtain

$$
\begin{aligned}
& \|F(u, \lambda)-F(v, \lambda)\| \\
& \leq\left[\sqrt{1-2 \rho \alpha+(p-1) \rho^{2} \beta^{2}}+\rho \xi+\mu\right]\|u-v\| \\
& =\theta\|u-v\|,
\end{aligned}
$$

where $\theta=\sqrt{1-2 \rho \alpha+(p-1) \rho^{2} \beta^{2}}+\rho \xi+\mu$. It follows from condition (3.3) that $\theta<1$. That is, $F(u, \lambda)$ is a contraction mapping which is uniform with respect to $\lambda \in \Omega$.

(ii) Since $F(u, \lambda)$ is a uniform contractive mapping with respect to $\lambda \in \Omega$, by the Banach fixed point theorem, $F(x, \lambda)$ has a unique fixed point $u(\lambda)$ for each $\lambda \in \Omega$. By Theorem 3.1, $S(\lambda) \neq \phi$.

For each $\lambda \in \Omega$, let $\left\{u_{n}\right\} \subset S(\lambda)$ and $u_{n} \rightarrow u_{0}$ as $n \rightarrow \infty$. Then we have

$$
u_{n}=F\left(u_{n}, \lambda\right), \quad n=1,2, \cdots .
$$

By (i), we have

$$
\left\|F\left(u_{n}, \lambda\right)-F\left(u_{0}, \lambda\right)\right\| \leq \theta\left\|u_{n}-u_{0}\right\| .
$$

It follows that 


$$
\begin{aligned}
\left\|u_{0}-F\left(u_{0}, \lambda\right)\right\| \leq & \left\|u_{0}-u_{n}\right\|+\left\|u_{n}-F\left(u_{n}, \lambda\right)\right\| \\
& +\left\|F\left(u_{n}, \lambda\right)-F\left(u_{0}, \lambda\right)\right\| \\
\leq & (1+\theta)\left\|u_{n}-u_{0}\right\| \rightarrow 0 \text { as } n \rightarrow \infty .
\end{aligned}
$$

Hence, we have $u_{0}=F\left(u_{0}, \lambda\right)$ and $u_{0} \in S(\lambda)$. Therefore, $S(\lambda)$ is a nonempty closed subset of $E$.

Remark 3.1. Theorem 3.2 is an improvement of Lemma 3.1 in $[2,17]$ from Hilbert spaces to $L_{p} \operatorname{spaces}(p \geq 2)$.

Theorem 3.3. Under the hypotheses of Theorem 3.2, further assume that for any $u, v, w \in E$, the mappings $\lambda \rightarrow N(u, v, \lambda)$ and $\lambda \rightarrow R_{\rho}^{M(\cdot, u, \lambda)}(w)$ are both Lipschitz continuous from $\Omega$ to $E$ with Lipschitz constants $l_{N}$ and $l_{R}$, respectively. Then the solution $z(\lambda)$ of the problem (2.1) is Lipschitz continuous from $\Omega$ to $E$.

Proof. For each $\lambda, \bar{\lambda} \in \Omega$, by Theorem 3.2 and the definition of $F(u, \lambda)$, we have

$$
\begin{aligned}
\|z(\lambda)-z(\bar{\lambda})\| & =\|F(z(\lambda), \lambda)-F(z(\bar{\lambda}), \bar{\lambda})\| \\
& \leq\|F(z(\lambda), \lambda)-F(z(\bar{\lambda}), \lambda)\|+\|F(z(\bar{\lambda}), \lambda)-F(z(\bar{\lambda}), \bar{\lambda})\| \\
& \leq \theta\|z(\lambda)-z(\bar{\lambda})\|+\|F(z(\bar{\lambda}), \lambda)-F(z(\bar{\lambda}), \bar{\lambda})\|
\end{aligned}
$$

and

$$
\begin{aligned}
& \|F(z(\bar{\lambda}), \lambda)-F(z(\bar{\lambda}), \bar{\lambda})\| \\
& =\left\|R_{\rho}^{M(\cdot, z(\bar{\lambda}), \lambda)}(z(\bar{\lambda})-\rho N(z(\bar{\lambda}), z(\bar{\lambda}), \lambda))-R_{\rho}^{M(\cdot, z(\bar{\lambda}), \bar{\lambda})}(z(\bar{\lambda})-\rho N(z(\bar{\lambda}), z(\bar{\lambda}), \bar{\lambda}))\right\| \\
& \leq\left\|R_{\rho}^{M(\cdot, z(\bar{\lambda}), \lambda)}(z(\bar{\lambda})-\rho N(z(\bar{\lambda}), z(\bar{\lambda}), \lambda))-R_{\rho}^{M(\cdot, z(\bar{\lambda}), \lambda)}(z(\bar{\lambda})-\rho N(z(\bar{\lambda}), z(\bar{\lambda}), \bar{\lambda}))\right\| \\
& \quad+\left\|R_{\rho}^{M(\cdot, z(\bar{\lambda}), \lambda)}(z(\bar{\lambda})-\rho N(z(\bar{\lambda}), z(\bar{\lambda}), \bar{\lambda}))-R_{\rho}^{M(\cdot, z(\bar{\lambda}), \bar{\lambda})}(z(\bar{\lambda})-\rho N(z(\bar{\lambda}), z(\bar{\lambda}), \bar{\lambda}))\right\| \\
& \leq\left(\rho l_{N}+l_{R}\right)\|\lambda-\bar{\lambda}\| .
\end{aligned}
$$

It follows from (3.7) and (3.8) that

$$
\|z(\lambda)-z(\bar{\lambda})\| \leq \frac{\rho l_{N}+l_{R}}{1-\theta}\|\lambda-\bar{\lambda}\| .
$$

This proves that $z(\lambda)$ is Lipschitz continuous in $\lambda \in \Omega$.

\section{REFERENCES}

[1] S.Adly, Perturbed algorithms and sensitivity analysis for a general class of variational inclusions, J. Math. Anal. Appl., 201 (1996), 609-630. 
[2] R. P. Agarwal, Y. J. Cho and N. J. Huang, Sensitivity analysis for strongly nonlinear quasivariational inclusions, Appl. Math. Lett., 13(2) (2000), 19-24.

[3] V. Barbu, Nonlinear Semigroups and Differential Equations in Banach spaces, Noordhoff Internat. Publ. Leyden, The Netherlands (1976).

[4] C. E. Chidume, Iterative solution of nonlinear equations of the monotone and dissipative type, Appl. Anal., 33 (1989), 79-86.

[5] C. E. Chidume, An iterative process for nonlinear Lipschitzian strongly accretive mappings in $L_{p}$ spaces, J. Math. Anal. Appl., 151 (2) (1990), 453-461.

[6] S. Dafermos, Sensitivity analysis in variational inequalities, Mathematics of Operators Research, 13 (1998), 421-434.

[7] X. P. Ding, Perturbed proximal point algorithm for generalized quasivariational inequalities, J. Math. Anal. Appl., 210 (1997), 88-101.

[8] X. P. Ding and C. L. Luo, On parametric generalized quasivariational inequalities, J. Optim. Theory and Appl., 100(1) (1999), 195-205.

[9] A. Hassouni and A. Moudafi, A perturbed algorithm for variational inclusions, J. Math. Anal. Appl., 185 (1994), 706-721.

[10] N. J. Huang, Mann and Ishikawa type perturbed iterative algorithms for nonlinear implicit quasivariational inclusions, Computers Math. Appl., 35(10) (1998), 9-14.

[11] K. R. Kazmi, Mann and Ishikawa perturbed iterative algorithms for generalized quasivariational inclusions, J. Math. Anal. Appl., 35(10) (1998), 9-14.

[12] Shu Jie Li and De Xing Feng, The topological degree for multivalued maximal monotone operator in Hilbert spaces, Acta math. Sinica, 25(5) (1982), 533-541.

[13] R. N. Mukherjee and H. L. Verma, Sensitivity analysis of generalized variational inequalities, J. Math. Anal. Appl., 167 (1992), 299-304.

[14] M. A. Noor, General algorithm and sensitivity analysis for variational inequalities, Journal of Applied Mathematics and Stochastic Analysis, 5 (1992), 29-42.

[15] M. A. Noor, Generalized set-valued variational inclusions and resolvent equations, J. Math. Anal. Appl., 228 (1998), 206-220.

[16] M. A. Noor, K. I. Noor and T. M. Rassias, Set-valued resolvent equations and mixed variational inequalities, J. Math. Anal. Appl., 220 (1998), 741-759.

[17] M. A. Noor and K. I. Noor, Sensitivity analysis for quasi-variational inclusions, J. Math. Anal. Appl., 236 (1999), 290-299.

[18] Y. H. Pan, Sensitivity analysis for general quasivariational inequalities, Journal of the Sichuan Normal University, 19 (1996), 56-59.

[19] J. Y. Park and J. U. Jeong, Parametric generalized mixed variational inequalities, Applied Mathematics Letters, 17 (2004), 43-48.

[20] N. D. Yen, Lipschitz continuity of solution of variational inequalities with a paramtric polyhedral constraint, Mathematics of Operations Research, 20 (1995), 695-708.

\section{Received: July 2, 2005}

